\title{
Control of Non-Adiabatic Passage through a Conical Intersection by a Dynamic Resonance
}

\author{
Michael Epshtein, Yair Yifrach, Alexander Portnov and Ilana Bar ${ }^{*}$ \\ Department of Physics, Ben-Gurion University of the Negev, Beer-Sheva 84105, Israel.
}

\section{Contents}

1. The model for analysis of the $\widetilde{A} \leftarrow \widetilde{X}$ excitation spectrum of $\mathrm{CH}_{3} \mathrm{ND} \quad \mathrm{S} 2$

1.1 Figure S1. Measured and simulated spectra of the origin and $v_{9}$ bands of $\mathrm{CH}_{3} \mathrm{ND}_{2}$ in the $\widetilde{A} \leftarrow \widetilde{X}$ transition S5

1.2 Table S1. Calculated frequencies of the transitions to $v_{9} \quad$ S6

2. Total kinetic energy distributions of the $D$ photofragments and internal $\begin{array}{ll}\text { energies of the } \mathrm{CH}_{3} \mathrm{ND} \text { radical } & \mathrm{S} 7\end{array}$

2.1 Figure S2. Kinetic energy distributions of D photofragments from S8 predissociation of $\mathrm{CH}_{3} \mathrm{ND}_{2}$ molecules excited to the $0_{0}^{0}$ and $v_{9}$ bands

2.2 Table S2. Retrieved internal energy of the ensuing $\mathrm{CH}_{3} \mathrm{ND}$ radical S9 $\begin{array}{lr}\text { References } & \text { S10 }\end{array}$ 


\section{The model for analysis of the $\widetilde{A} \leftarrow \widetilde{X}$ excitation spectrum of $\mathbf{C H}_{3} \mathbf{N D}_{2}$}

The simulation of the spectra of $\mathrm{CH}_{3} \mathrm{ND}_{2}$ in the regions of the $0_{0}^{0}$ and $v_{9}$ state was performed while adopting the model of Kim and coworkers. ${ }^{1,2,3}$ Briefly, two types of Hamiltonians, namely an asymmetric rigid rotor Hamiltonian ${ }^{4}$ for the electronic ground state and another one, based on the symmetric rotor approximation with the addition of internal molecular rotation of the two moieties, i.e., the $\mathrm{CH}_{3}$ with respect to $\mathrm{ND}_{2}$, for the excited electronic state were used. The asymmetric rigid rotor Hamiltonian is given by: ${ }^{4}$

$$
\mathrm{H}_{\mathrm{r}}=a J_{a}^{2}+b J_{b}^{2}+c J_{c}^{2}
$$

and can be rewritten as,

$$
\mathrm{H}_{\mathrm{r}}=\frac{1}{2}(a+b)\left(J_{a}^{2}+J_{b}^{2}\right)+c J_{c}^{2}+\frac{1}{2}(a-b)\left(J_{a}^{2}-J_{b}^{2}\right)
$$

where the set $\mathrm{a}, \mathrm{b}$ and $\mathrm{c}$ maps the rotational constants $\mathrm{A}, \mathrm{B}$ and $\mathrm{C}$ of the molecule and the $\mathrm{J}_{\mathrm{i}} \mathrm{s}$ are the angular momenta with respect to the principal axes of the molecule.

Equation (2) was rewritten in the following form:

$$
\mathrm{H}_{\mathrm{r}}=\alpha \boldsymbol{J}^{2}+\beta J_{c}^{2}+\gamma\left(J^{+2}+J^{-2}\right)
$$

where,

$$
\alpha=\frac{1}{2}(a+b), \beta=c-\frac{1}{2}(a+b), \gamma=\frac{1}{4}(a-b) .
$$

The mapping set chosen was:

$$
\mathrm{a}=\mathrm{B}, \mathrm{b}=\mathrm{C}, \mathrm{c}=\mathrm{A} \text {, }
$$

The Hamiltonian was constructed using the eigenstates for the symmetric top:

$$
<J K\left|J^{2}\right| J K>=J(J+1)
$$




$$
\begin{aligned}
& <J K\left|J_{c}^{2}\right| J K>=K^{2} \\
& <J K+2\left|J^{-} J^{-}\right| J K>=<J K+2\left|J^{+} J^{+}\right| J K+2>=[(J-K)(J+K+1)(J-K-1)(J+ \\
& K+2)]^{\frac{1}{2}} \\
& <J K-2\left|J^{+} J^{+}\right| J K>=<J K+2\left|J^{-} J^{-}\right| J K-2>=[(J+K)(J-K+1)(J+K-1)(J- \\
& K+2)]^{\frac{1}{2}}
\end{aligned}
$$

This Hamiltonian was diagonalized, with its eigenvalues sorted in descending order and characterized by three quantum numbers i.e., $J, K_{A}, K_{C}$, where $K_{A}$, and $K_{C}$ are the absolute values of the typical projections for the limiting prolate and oblate symmetric rotor cases, respectively.

The Hamiltonian for the excited electronic state is given by: ${ }^{\mathbf{1 , 2 , 3}}$

$$
\begin{aligned}
& H=H_{\text {rot }}+F(p-P)^{2}+V(\phi), \\
& V(\phi)=\left(V_{6} / 2\right)[1-\cos (6 \phi)],
\end{aligned}
$$

where $H_{\text {rot }}$, is the standard rigid-rotor symmetric-top approximated rotational Hamiltonian, $F$ is an effective rotational constant for the internal rotation, $V_{6}$ is the torsional barrier height of a six-fold torsional potential energy surface along the torsional angle $\phi$ and the $(p-P)$ term is the relative angular momentum of the top and frame.

The free-rotor basis set $\left\{\mid m>=(2 \pi)^{-1 / 2} \exp (-i m \phi)\right\}$ was used to construct the Hamiltonian matrix and the nonvanishing matrix elements are given by:

$$
\begin{gathered}
<m|H| m>=2 A^{\prime} m^{2}+B^{\prime} J^{\prime}\left(J^{\prime}+1\right)+\left(A^{\prime}-B^{\prime}\right) K^{\prime 2}-2 A^{\prime} m K^{\prime}+\left(V_{6} / 2\right) \\
<m|H| m \pm 6>=-\left(V_{6} / 4\right)
\end{gathered}
$$


where $A^{\prime}$ is twice the rotational constant along the $\mathrm{C}-\mathrm{N}$ bond and $B^{\prime}$ is the other rotational constant of the symmetric top. Also, $b J^{\prime}$ is the total angular momentum quantum number and $K^{\prime}$ is the quantum number representing the projection of the angular momentum on the C-N bond axis. The Hamiltonian in Eqs. (12-13) was diagonalized. Also, the intensities of the lines were calculated, while accounting for the Boltzmann distribution of the rotational levels in the vibrationless ground state and the spin statistics of the transitions and arbitrary factoring the different $m$ values of the excited state.

Based on the above Hamiltonians and the factors governing the intensities, a model was built and a Matlab code was written to obtain the calculated spectrum, by using the rotational constants and linewidths given by Kim and co-workers ${ }^{1}$ and assuming a rotational temperature of $15 \mathrm{~K}$.

As can be seen from Figure S1 the (a) measured and (b) simulated spectra of the origin, $0_{0}^{0}$, and $v_{9}$ bands of $\mathrm{CH}_{3} \mathrm{ND}_{2}$ in the $\widetilde{A} \leftarrow \tilde{X}\left(\mathrm{~S}_{1} \leftarrow \mathrm{S}_{0}\right)$ transition match well, allowing, based on the results shown in Table S1 to identify the different transitions involved in each line, and the populations of the different contributing rotational states. 


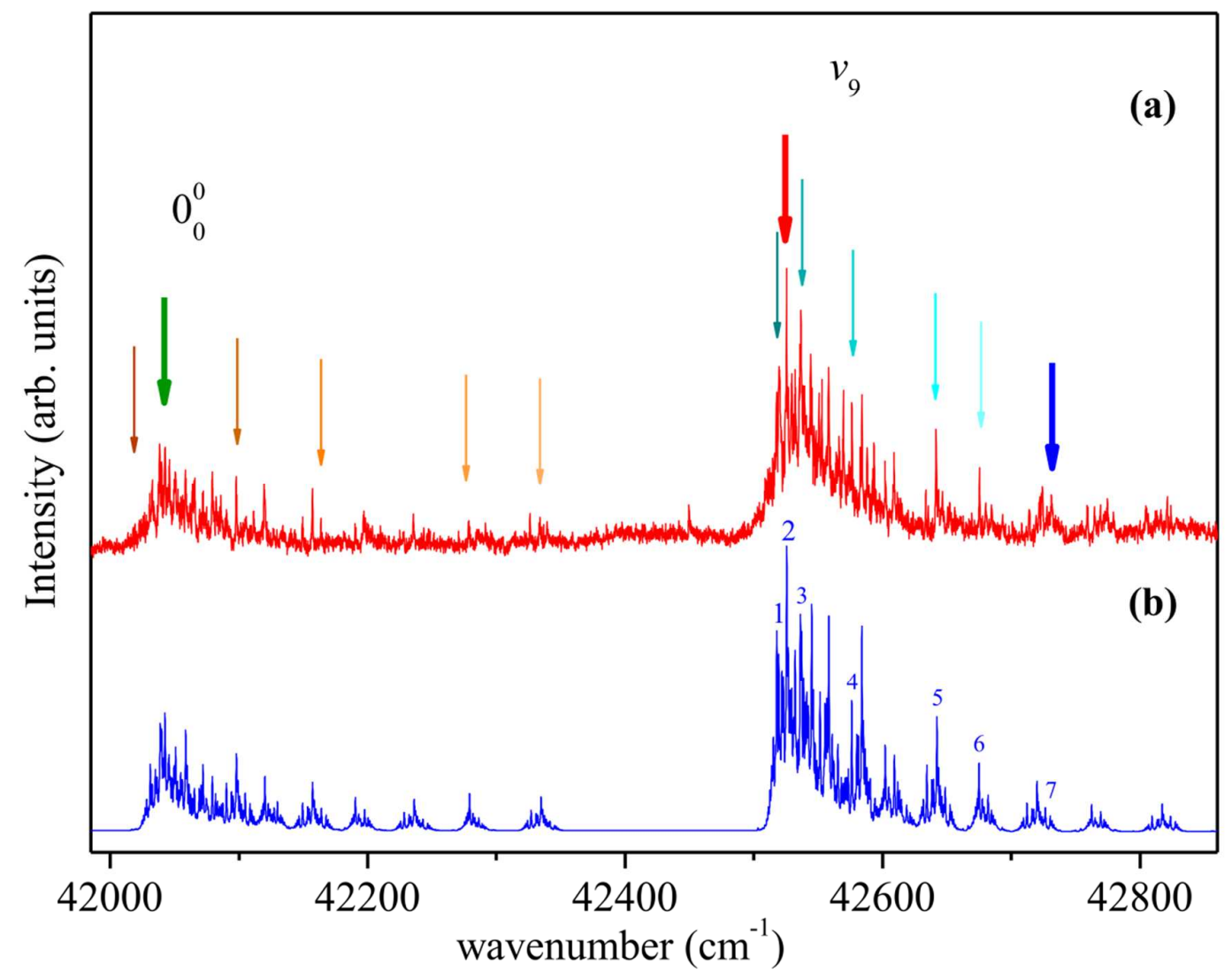

Figure S1. (a) Measured and (b) simulated spectra of the origin, $0_{0}^{0}$, and $v_{9}$ bands of $\mathrm{CH}_{3} \mathrm{ND}_{2}$ in the $\tilde{A} \leftarrow \tilde{X}\left(\mathrm{~S}_{1} \leftarrow \mathrm{S}_{0}\right)$ transition. The simulation is based on the above described Hamiltonians. The arrows, above the measured spectrum, mark the transitions that were used for monitoring the two-color reduced-Doppler velocity map images of the D photofragments [see Figure 1(a)]. The numbers above the simulated spectrum, mark the transitions in Table S1. 
Table S1. Calculated frequencies of the transitions from rotations of the vibrationless ground state to those on $v_{9}$ on the excited electronic state, together with the angular momentum quantum numbers and the calculated frequencies of ground and excited states, the population in the different rotational states on the ground state involved in the transitions and the observed - calculated frequencies.

\begin{tabular}{|c|c|c|c|c|c|c|c|c|c|c|c|}
\hline & \multirow{2}{*}{$\Delta E\left(\mathrm{~cm}^{-1}\right)^{a}$} & \multicolumn{4}{|c|}{ Excited states } & \multicolumn{4}{|c|}{ Ground states } & \multirow{2}{*}{$\begin{array}{c}\text { Population } \\
(\%)\end{array}$} & \multirow{2}{*}{$\begin{array}{c}\text { Obs - Calc } \\
\left(\mathrm{cm}^{-1}\right)\end{array}$} \\
\hline & & $\mathrm{J}^{\prime}$ & $\mathrm{K}^{\prime}$ & $\mathrm{m}^{\prime}$ & $E^{\prime}\left(\mathrm{cm}^{-1}\right)$ & J" & $\mathrm{K}_{\mathrm{a}}^{\prime \prime}$ & $\mathrm{K}_{\mathrm{c}}{ }^{\prime \prime}$ & $E^{\prime \prime}\left(\mathrm{cm}^{-1}\right)$ & & \\
\hline \multirow{4}{*}{1} & 42522.5 & 2 & 0 & 0 & 13.5 & 2 & 1 & 1 & 5.9 & 57 & 0.6 \\
\hline & 42522.5 & 1 & 0 & 0 & 10.8 & 1 & 1 & 0 & 3.3 & 73 & 0.6 \\
\hline & 42522.6 & 1 & 0 & 0 & 10.8 & 1 & 1 & 1 & 3.2 & 73 & 0.5 \\
\hline & 42522.7 & 2 & 0 & 0 & 13.5 & 2 & 1 & 2 & 5.8 & 57 & 0.4 \\
\hline \multirow{6}{*}{2} & 42528.7 & 1 & 1 & 0 & 15.0 & 1 & 0 & 1 & 1.3 & 88 & 0.0 \\
\hline & 42528.7 & 1 & 1 & 1 & 15.0 & 1 & 0 & 1 & 1.3 & 88 & 0.0 \\
\hline & 42528.7 & 2 & 1 & 1 & 17.6 & 2 & 0 & 2 & 3.9 & 69 & 0.0 \\
\hline & 42528.7 & 2 & 1 & 0 & 17.6 & 2 & 0 & 2 & 3.9 & 69 & 0.0 \\
\hline & 42528.8 & 3 & 1 & 1 & 21.6 & 3 & 0 & 3 & 7.8 & 47 & -0.1 \\
\hline & 42528.8 & 3 & 1 & 0 & 21.6 & 3 & 0 & 3 & 7.8 & 47 & -0.1 \\
\hline \multirow{6}{*}{3} & 42539.3 & 3 & 2 & 0 & 34.2 & 3 & 1 & 2 & 9.9 & 39 & 0.5 \\
\hline & 42539.3 & 3 & 2 & 2 & 34.2 & 3 & 1 & 2 & 9.9 & 39 & 0.5 \\
\hline & 42539.3 & 2 & 2 & 2 & 30.2 & 2 & 1 & 1 & 5.9 & 56 & 0.5 \\
\hline & 42539.3 & 2 & 2 & 0 & 30.2 & 2 & 1 & 1 & 5.9 & 56 & 0.5 \\
\hline & 42539.5 & 2 & 2 & 2 & 30.3 & 2 & 1 & 2 & 5.8 & 57 & 0.4 \\
\hline & 42539.5 & 2 & 2 & 0 & 30.3 & 2 & 1 & 2 & 5.8 & 57 & 0.4 \\
\hline \multirow{4}{*}{4} & 42579.2 & 2 & 1 & 3 & 76.0 & 2 & 2 & 0 & 11.7 & 32 & 0.2 \\
\hline & 42579.2 & 2 & 1 & -2 & 76.0 & 2 & 2 & 0 & 11.7 & 32 & 0.2 \\
\hline & 42579.2 & 2 & 1 & 3 & 76.0 & 2 & 2 & 1 & 11.7 & 32 & 0.2 \\
\hline & 42579.2 & 2 & 1 & -2 & 76.0 & 2 & 2 & 1 & 11.7 & 32 & 0.2 \\
\hline \multirow{6}{*}{5} & 42645.3 & 1 & 1 & -3 & 131.7 & 1 & 0 & 1 & 1.3 & 88 & -0.6 \\
\hline & 42645.3 & 1 & 1 & 4 & 131.7 & 1 & 0 & 1 & 1.3 & 88 & -0.6 \\
\hline & 42645.4 & 2 & 1 & -3 & 134.3 & 2 & 0 & 2 & 3.9 & 69 & -0.6 \\
\hline & 42645.4 & 2 & 1 & 4 & 134.3 & 2 & 0 & 2 & 3.9 & 69 & -0.6 \\
\hline & 42645.4 & 3 & 1 & 4 & 138.2 & 3 & 0 & 3 & 7.8 & 47 & -0.7 \\
\hline & 42645.4 & 3 & 1 & -3 & 138.2 & 3 & 0 & 3 & 7.8 & 47 & -0.7 \\
\hline \multirow{6}{*}{6} & 42678.0 & 2 & 0 & -4 & 169.0 & 2 & 1 & 1 & 5.9 & 57 & 0.5 \\
\hline & 42678.0 & 2 & 0 & 4 & 169.0 & 2 & 1 & 1 & 5.9 & 57 & 0.5 \\
\hline & 42678.0 & 1 & 0 & -4 & 166.3 & 1 & 1 & 0 & 3.3 & 73 & 0.5 \\
\hline & 42678.0 & 1 & 0 & 4 & 166.3 & 1 & 1 & 0 & 3.3 & 73 & 0.5 \\
\hline & 42678.1 & 1 & 0 & -4 & 166.3 & 1 & 1 & 1 & 3.2 & 73 & 0.4 \\
\hline & 42678.1 & 1 & 0 & 4 & 166.3 & 1 & 1 & 1 & 3.2 & 73 & 0.4 \\
\hline \multirow{2}{*}{7} & 42733.5 & 3 & 3 & -3 & 230.2 & 2 & 2 & 0 & 11.7 & 32 & 1.0 \\
\hline & 42733.5 & 3 & 3 & -3 & 230.2 & 2 & 2 & 1 & 11.7 & 32 & 1.0 \\
\hline
\end{tabular}


${ }^{a} \Delta E=E^{\prime}-E^{\prime \prime}+E_{\mathrm{vib}}\left(v_{9}\right)+T_{0}$, where $E_{\mathrm{vib}}\left(v_{9}\right)=480 \mathrm{~cm}^{-1}$ and $T_{0}=42035 \mathrm{~cm}^{-1} . T_{0}$ and $E_{\mathrm{vib}}$ were taken from Ref. 1.

\section{Total kinetic energy distributions of the $D$ photofragments and internal energies of the $\mathrm{CH}_{3} \mathrm{ND}$ radical}

The total kinetic energy distributions (TKEDs) of the $\mathrm{CH}_{3} \mathrm{ND}+\mathrm{D}$ photofragments displayed in Figure 3 show representative curves, resulting from predissociation of $\mathrm{CH}_{3} \mathrm{ND}_{2}$ molecules promoted to different rovibronic states on the $\widetilde{A} \leftarrow \widetilde{X}$ transition. Here in Figures $\mathrm{S} 2(\mathrm{a})$ and $\mathrm{S} 2(\mathrm{~b})$, the focus is on TKEDs resulting from predissociation of molecules to the rovibronic states of the $0_{0}^{0}$ and $v_{9}$ bands. It can be seen that in the former the distributions almost overlap, while in the latter there is a change in the extent of production of slow D photofragments and a shift in the maximal translational energy, where the distribution that corresponds to the maximal point in the dynamic resonance is the one displayed with the darkest cyan [Figure S2(b)]. Also shown in Figure S2(a) is a vertical line that cuts the darkest brown distribution, corresponding to the first measured rovibronic state in the origin band, at an intensity of 0.5 of the falling edge. This point allowed to determine the N-D bond energy and similarly the maximal translational energies for all the other measured TKEDs from the different rovibronic states. These results are summarized in Table S2. 


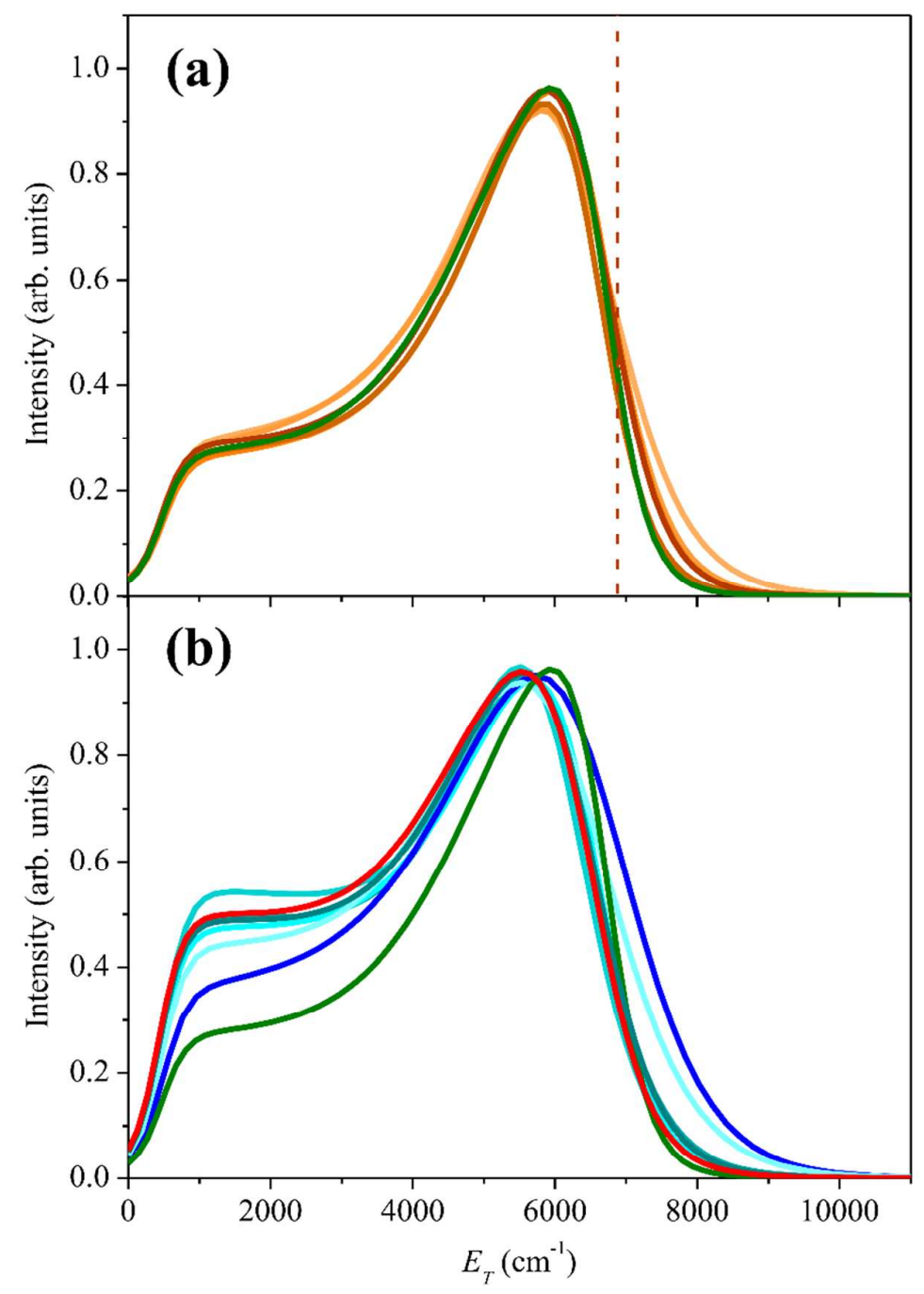

Figure S2. The fitted total kinetic energy distributions of the $\mathrm{CH}_{3} \mathrm{ND}+\mathrm{D}$ photofragments, resulting from predissociation of $\mathrm{CH}_{3} \mathrm{ND}_{2}$ molecules, promoted to the rovibronic states of (a) $0_{0}^{0}$ [marked by green and brown color arrows in Figure 1(d) and in Figure S1] and of (b) $v_{9}$ [marked by red, blue and cyan arrows in Figure 1(d) and in Figure S1]. Panel (b) also includes the green distribution corresponding to the second rovibronic transition of $0_{0}^{0}$ state. The dashed vertical line in (a) shows the point of intersection between the line and the darker brown distribution from which the bond energy was determined. 
Table S2. The energies of the predissociating photon, the maximal translational energies, the available energies and the internal energy of the ensuing radical obtained in the predissociation of $\mathrm{CH}_{3} \mathrm{ND}_{2}$ excited to different rovibronic states.

\begin{tabular}{|c|c|c|c|c|}
\hline & $E_{h v}\left(\mathrm{~cm}^{-1}\right)^{a}$ & $E_{T}\left(\mathrm{~cm}^{-1}\right)^{b}$ & $E_{a v l}\left(\mathrm{~cm}^{-1}\right)^{c}$ & $\begin{array}{c}E_{\text {int }}\left(\mathrm{CH}_{3} \mathrm{ND}\right) \\
\left(\mathrm{cm}^{-1}\right)^{d}\end{array}$ \\
\hline \multirow{6}{*}{$0_{0}^{0}$} & 42021 & 6870 & 6870 & 0 \\
\hline & 42049 & 6760 & 6900 & 140 \\
\hline & 42101 & 6720 & 6950 & 230 \\
\hline & 42167 & 6880 & 7010 & 130 \\
\hline & 42282 & 6850 & 7130 & 280 \\
\hline & 42336 & 6890 & 7180 & 290 \\
\hline \multirow{7}{*}{$v_{9}$} & 42523 & 6650 & 7370 & 720 \\
\hline & 42529 & 6590 & 7380 & 790 \\
\hline & 42540 & 6650 & 7390 & 740 \\
\hline & 42579 & 6550 & 7430 & 880 \\
\hline & 42645 & 6690 & 7490 & 800 \\
\hline & 42679 & 6870 & 7530 & 660 \\
\hline & 42735 & 7130 & 7580 & 450 \\
\hline $2 v_{9}$ & 43029 & 7740 & 7880 & 140 \\
\hline$v_{7}$ & 43051 & 7700 & 7900 & 200 \\
\hline $3 v_{9}$ & 43536 & 7840 & 8380 & 550 \\
\hline$v_{7}+v_{9}$ & 43562 & 7950 & 8410 & 460 \\
\hline $4 v_{9}$ & 44036 & 8180 & 8880 & 700 \\
\hline $2 v_{7}$ & 44085 & 8160 & 8930 & 770 \\
\hline$v_{7}+2 v_{9}$ & 44127 & 8010 & 8970 & 960 \\
\hline $2 v_{7}+v_{9}$ & 44553 & 8230 & 9400 & 1170 \\
\hline$v_{7}+3 v_{9}$ & 44622 & 8350 & 9470 & 1120 \\
\hline $3 v_{7}$ & 45085 & 8280 & 9930 & 1650 \\
\hline $2 v_{7}+2 v_{9}$ & 45165 & 8470 & 10010 & 1540 \\
\hline
\end{tabular}

${ }^{a} E_{h v}$ - energy of the predissociating photon

${ }^{b} E_{T}$ - maximal total translational energy, i.e., the energy at half height of the falling edge

${ }^{c} E_{a v l}=E_{h v}-D_{0}(\mathrm{~N}-\mathrm{D})$

${ }^{d} E_{\text {int }}\left(\mathrm{CH}_{3} \mathrm{ND}\right)$ - internal energy of the $\mathrm{CH}_{3} \mathrm{ND}$ photofragments $E_{\text {int }}\left(\mathrm{CH}_{3} \mathrm{ND}\right) \approx E_{\text {avl }}-E_{T}$.

The inaccuracy in $E_{T}$ and $E_{a v l}$ is $\pm 400 \mathrm{~cm}^{-1}$ and that of $E_{\text {int }}\left(\mathrm{CH}_{3} \mathrm{ND}\right)$, relative to ground state, $\pm 100 \mathrm{~cm}^{-1}$. The inaccuracy in $E_{T}$ was calculated based on ref. 5, to account for the recoil momentum imparted to the $\mathrm{D}^{+}$ion by the photoelectron during the probing of $\mathrm{D}$ atoms by the $(2+1)$ resonantly enhanced multiphoton ionization process as well as for the phosphor screen resolution and instrumental error. 


\section{References}

${ }^{1}$ Park, M. H.; Choi, K.-W.; Choi, Y. S.; Kim, S. K. Vibrational Structures of Methylamine Isotopomers in the Predissociative $\widetilde{A}$ states: $\mathrm{CH}_{3} \mathrm{NHD}, \mathrm{CD}_{3} \mathrm{NH}_{2}, \mathrm{CD}_{3} \mathrm{NHD}$, and $\mathrm{CD}_{3} \mathrm{ND}_{2} . J$. Chem. Phys. 2006, 125, 084311.

${ }^{2}$ Baek, S. J.; Choi, K. -W.; Choi, Y. S.; Kim, S. K. Spectroscopy and Dynamics of Methylamine. I. Rotational and Vibrational Structures of $\mathrm{CH}_{3} \mathrm{NH}_{2}$ and $\mathrm{CH}_{3} \mathrm{ND}_{2}$ in $\widetilde{A}$ States. J. Chem. Phys. 2003, 118, 11026-11039.

${ }^{3}$ Baek, S. J.; Choi, K. -W.; Choi, Y. S.; Kim, S. K. Vibrational Structures of Predissociating Methylamines $\left(\mathrm{CH}_{3} \mathrm{NH}_{2}\right.$ and $\left.\mathrm{CH}_{3} \mathrm{ND}_{2}\right)$ in $\widetilde{A}$ States: Free Internal Rotation of $\mathrm{CH}_{3}$ with Respect to $\mathrm{NH}_{2}$. J. Chem. Phys. 2002, 117, 10057-10060.

${ }^{4}$ Kroto, H. W. Molecular Rotation Spectra, John Wiley \& Sons, London (1975).

${ }^{5}$ Toomes, R.L.; Samartzis, P. C.; Rakitzis, T. P.; Kitsopoulos, T. N. Slice Imaging of H-Atom Photofragments: Effects of the REMPI Detection Process on the Observed Velocity Distribution. Chem. Phys. 2004, 301, 209-212. 